\title{
Variations
}

Variations

Revue internationale de théorie critique

$17 \mid 2012$

Critique du travail

\section{Éviter que ne soit retenu comme coupable le travail lui-même}

Didier Epsztajn

\section{(2) OpenEdition}

Journals

Édition électronique

URL : http://journals.openedition.org/variations/428

DOI : 10.4000/variations.428

ISSN : 1968-3960

Éditeur

Les amis de Variations

Référence électronique

Didier Epsztajn, «Éviter que ne soit retenu comme coupable le travail lui-même », Variations [En ligne],

17 | 2012, mis en ligne le 15 octobre 2012, consulté le 22 septembre 2020. URL : http://

journals.openedition.org/variations/428; DOI : https://doi.org/10.4000/variations.428

Ce document a été généré automatiquement le 22 septembre 2020.

Les ami•e•s de Variations 


\title{
Éviter que ne soit retenu comme coupable le travail lui-même
}

\author{
Didier Epsztajn
}

\section{RÉFÉRENCE}

Isabelle Forno, Travail, peurs et résistances. Critique de la victimisation du salarié, Editions Syllepse, Paris 2012, 148 pages, 15 euros

\section{NOTE DE L'ÉDITEUR}

Une première version de cette recension est parue le 2 avril 2012 sur le site Entre les lignes entre les mots

http://entreleslignesentrelesmots.wordpress.com/2012/04/02/eviter-que-ne-soitretenu-comme-coupable-le-travail-lui-meme/

Se tenir sur le fil du rasoir comme un danseur de cordes, repérer depuis un point à la fois menacé et invincible, les périls qui guettent de toutes parts et les chances qui gisent à même le danger, telle est à la fois la ligne droite et sinueuse de la résistance. (Françoise Proust, citée par l'auteure.)

1 L'auteure, qui a exercé durant trente ans des fonctions de direction des mal-nommées ressources humaines, nous livre ici ses "premiers mots de femme libre ». Son expérience et ses réflexions donnent lieu à un livre réjouissant, au langage sans détours, acerbe et ironique, en décalage avec les propos souvent tenus jusque dans les milieux syndicaux sur la « souffrance au travail ».

Dans le système capitaliste mondialisé, l'entreprise est au centre de tout, l'entreprise et ses actionnaires qui, soi-disant, prennent tous les risques face à la concurrence. Un conte pas très moral pour masquer tout un pan de réalités pourtant bien tangibles. Exit les salarié-e-s, exit la subordination, exit les résistances, "exit (...) la question de la conflictualité autour du travail, de son organisation, de son sens ». Place à la " responsabilité 
sociale» et à la "surveillance des risques psychosociaux». Cette présentation des choses très en vogue vise à transformer, ou du moins à maquiller le cœur du rapport salarial et la figure du salarié :

Le statufiant en quelque sorte sous la posture du " gisant ", elle l'implore de garder sagement la pose, pendant que le travail, principale source de la souffrance salariale, continue de distiller ses effets ravageurs et de dysfonctionner en toute impunité. (p. 8)

Pourtant, "lorsqu'un suicide est reconnu comme accident du travail c'est finalement toute une société qui reconnaît que potentiellement travailler est un risque mortel. ${ }^{1}{ }^{~}$ Tout au long de ce livre divisé en quatre chapitres, centrés tout à tour sur " un salarié en souffrance(s) », " en méfiance(s)", "sous surveillance(s) » et "en résistance(s)", l'auteure nous invite donc à briser ce piège de la "victimisation d'autant plus pervers qu'en cernant les fragiles, il protège avant tout les fauteurs de troubles».

$\mathrm{Au}$ commencement bien évidemment se trouve la subordination :

Dans les relations de travail, la question de la subordination reste un élément central de la définition du salariat et structure à mon sens fortement le cadre juridique, mais également psychique, des relations entre un employeur et «son » subordonné. ${ }^{2}$. (p. 16)

L'auteure s'attache dans un premier moment à décrire les évolutions internes des entreprises, les nouvelles orientations du management, des réalités le plus souvent cachées :

Sous prétexte d'optimiser les organisations et de créer de la valeur, l'entreprise va imposer au travail toujours plus de sévices. Elle va le «tendre » (méthode kanban de gestion à flux tendu par exemple), le tronçonner (organisation en silo, externalisation des activités en dehors du cœur de métier), l'exiler (délocalisations), le trancher à vif (méthode du down sizing ou de suppression de postes), l'amaigrir (lean ou gestion drastique de ses frais), le harceler (kaizen : méthodes d'amélioration continue), l'écarteler (pression sur les objectifs, intensification de son rythme, et diminution de ses ressources). (pp. 89-90)

Et la/le salarié-e, rendu-e abstrait-e, est transformé-e en un «Équivalent Temps Plein en trop, autant dire un ennemi à éradiquer. »

Ces descriptions sont ensuite mises en regard avec le soin particulier que semblent développer les états-majors pour contenir les risques psychosociaux, à grands renforts de construction d'indicateurs - nouvel eldorado pour les cabinets de consulting et autres "Diafoirus des RPS ", mais où s'enlise littéralement l'action prétendument préventive :

L'obsession de la mesure semble trouver là sa caricature. [...] Comme s'il s'agissait [...] de passer un temps infini à la construction d'un thermomètre en en oubliant l'urgence du traitement et surtout, de penser que sans mesure il n'est nulle action efficace possible. (p. 69)

Il est donc plus que légitime de s'interroger frontalement comme le fait l'auteure. «Et si la fabrication du risque psychosocial avait pour fonction d'éviter de s'intéresser aux pathologies du travail, (...), et pour mieux l'innocenter, de victimiser le salarié?» Isabelle Forno rappelle ainsi à très juste titre que dans ce grand récit qui exclut les fondements de la réalité des salarié-e-s, le grand absent est... le travail lui-même. «Or pour les humains, le travail... ce n'est pas que le travail ». Les individu-es engagent en effet leur subjectivité et jouent leur puissance d'agir dans le travail qui, pour utiliser un langage que ne partagerait peutêtre pas l'auteure, est une forme historique de l'activité, particulière au système capitaliste, l'exploitation de la force de travail dans des rapports sociaux asymétriques. 
9 Mais d'après l'auteure, les salarié-e-s «en méfiance(s)» ne sont pas dupes de cette grande mise en scène de la lutte institutionnalisée contre les «risques psychosociaux »: "Comment accepter, en effet, d'être ainsi aidé et soigné par cette même entreprise qui vous met(trait) à mal, vous brutalise(rait), vous stresse(rait) ou vous harcèle(rait)?" Les salarié-e-s livrent en réalité une bataille quotidienne, faite de milliers d'actes infimes autant que de grands éclats, pour maintenir le travail vivant envers et contre sa rationalisation morbide. Je ne partage pas tout à fait les appréciations de l'auteure sur la disparition des formes de résistance ou de conflictualité «traditionnelles ». Certes les rapports de force entre groupes sociaux, la conscience individuelle de la situation et les formes d'organisation, plus ou moins collectives, fluctuent et évoluent, mais l'idée d'un monde du travail déserté par les luttes collectives relèvent plutôt d'une vision déformée de la réalité sociale ${ }^{3}$. L'auteure, elle, choisit de mettre l'accent sur les nouvelles formes de résistance et évoque nombre d'initiatives qu'elle a eu le loisir d'observer, des contre-blogs d'entreprises aux manifestations virtuelles (mais victorieuses !) sur Second Life $e^{\oplus}$, et dont la diversité et la multiplicité réjouissent :

Qu'il s'agisse de récalcitrance, de dissidence, de rébellion, de contestation ou, de l'autre coté de cet invisible bras de fer, de motivation, de coopération, de solidarité, nous assistons à une même déclinaison de ce que j'appellerai la «créativité des indignés. »

10 Cet ouvrage offre donc un regard décentré pour une critique de la victimisation des salarié-e-s et la remise au centre de la réflexion du travail, activité nuisible dans sa forme-même, qu'il convient, au-delà des adaptations nécessaires, de réduire à une durée la plus faible possible.

\section{NOTES}

1. Sur ce sujet, voir aussi le livre de la Fondation Copernic coordonné par Louis-Marie Barnier, Travailler tue en toute impunité, Editions Syllepse, Paris, 2009.

http://entreleslignesentrelesmots.wordpress.com/2010/05/18/la-delinquance-patronale-doitetre-sanctionnee/

2. Sur la subordination et plus généralement sur le droit du travail, voir aussi Laurent Willemez qui se demande «comment collectiviser un droit individualiste» dans Le droit du travail en danger. Une ressource collective pour des combats individuels, Editions du Croquant, Paris 2006.

http://entreleslignesentrelesmots.wordpress.com/2010/05/16/comment-collectiviser-un-droitindividualiste/

3. Voir par exemple l'ouvrage de Sophie Béroud, Jérôme Pélisse, Baptiste Giraud, Guillaume Desage, Jean-Michel Denis, La lutte continue? Les conflits du travail dans la France contemporaine, Editions du Croquant, Paris, 2008.

http://entreleslignesentrelesmots.wordpress.com/category/luttes/page/2/ 
INDEX

Mots-clés : critique du travail, correspondance, artistes, socialisme vert, travailleurs culturels, Wertkritik, Gorz André, dialogue, Vincent Jean-Marie, écologie, modèles alternatifs, Marx Karl, penser le Capital, ethos du travail, Eglise Orthodoxe, salariat, domination 